Radial and Nonradial Pulsations as Probes of Stellar Physics

ASP Conference Series, Vol. 259, 2002

C. Aerts, T.R. Bedding, \& J. Christensen-Dalsgaard, eds.

\title{
An Overview of Paul Ledoux' Work
}

\author{
P. Smeyers \\ Instituut voor Sterrenkunde, Katholieke Universiteit Leuven, Belgium
}

This IAU Colloquium on Radial and Nonradial Pulsations as Probes of Stellar Physics is dedicated to the memory of our former fellow countryman and former professor at the University of Liège, the astrophysicist Paul Ledoux. The opportunity is the fiftieth anniversary of the publication of his valuable paper on the effect of a small uniform axial rotation on nonradial stellar oscillations. The paper was published in 1951 in the Astrophysical Journal under the title "The nonradial oscillations of gaseous stars and the problem of Beta Canis Majoris" (Ledoux, 1951).

The paper resulted from a work Paul Ledoux had carried out during a stay at the Princeton University Observatory in 1950-1951. The idea of this work had occurred to him by a reading of a paper by Otto Struve (1950) on W.F. Meyer's study of the radial velocity of the star $\beta$ Canis Majoris.

Paul Ledoux' contribution can briefly be described as follows. In pulsation theory, the normal linear, adiabatic (isentropic) oscillation modes of a spherically symmetric star are expressed in terms of spherical harmonics of the colatitude and the azimuthal angle, both defined with respect to a frame of reference whose origin coincides with the mass centre of the star. The degree of a spherical harmonic is usually denoted by $\ell$, which is the notation adopted by Ledoux in his comprehensive review article on variable stars published in collaboration with Walraven in 1958 in the Handbuch der Physik (Ledoux \& Walraven, 1958); the azimuthal number of a spherical harmonic is commonly denoted by $m$. In a nonrotating spherically symmetric star, the eigenfrequencies of the nonradial oscillation modes of a degree $\ell$ and a given radial order are $(2 \ell+1)$-fold degenerate with respect to the azimuthal number $m$. In his paper of 1951, Paul Ledoux showed by means of a first-order perturbation method that, in a slowly uniformly rotating star, the Coriolis force lifts the degeneracy completely, so that the eigenfrequencies of the nonrotating star are splitted up into $(2 \ell+1)$ equidistant eigenfrequencies. With regard to the $\beta$ Canis Majoris stars, nowadays referred to as the $\beta$ Cephei stars, Paul Ledoux concluded that

the existence of two periods very close to each other as well as a phase shift of a quarter-period between the broadening of the lines and the corresponding radial velocity can be accounted for if the oscillation corresponds to a spherical harmonic of degree 2 in a rotating star.

Paul Ledoux' paper is remarkable also since it contained the seeds of various subsequent developments. One may think of three main developments. First of all, his theory has been the basis of a theory of magnetic variable stars he proposed in collaboration with Renson, in 1967, in terms of nonradial pulsations 
that are maintained in a superadiabatic region by the buoyancy force in presence of rotation, as well as by a toroidal magnetic field (Ledoux \& Renson, 1967).

Secondly, at Paul Ledoux' suggestion, the effects of a small uniform axial rotation on oscillation periods of stars have been determined up to the second order in the angular frequency of rotation, order at which the star's distortion due to the centrifugal force has to be taken into account. An appropriate perturbation procedure was developed by Simon (1969) for radial stellar oscillations and was extended by Smeyers \& Denis (1971) to nonradial stellar oscillations. Later, the effects of a small uniform axial rotation on oscillation periods of polytropic models were determined extensively by Saio (1981) (see also Unno et al. 1989, Section 19.2).

Parallel investigations have gone on into the effects of the distortion caused by an equilibrium tide (Denis, 1972; Saio, 1981). For several decades, these investigations were restricted to polytropic models. Recently, we have extended the procedure to arbitrary physical models of stars in collaboration with our graduate student T. Reyniers.

Thirdly, Ledoux' theory of nonradial oscillations for the phenomenon of the $\beta$ Cephei stars has been a starting-point for the identification of modes in variable stars from line profile variations in spectra. For this type of analysis, Ledoux' theory was usefully worked out by Osaki (1971). Balona, on his side, proposed the use of the moment method (Balona, 1986a, 1986b, 1987). The method was first applied to observed line profile variations of a $\beta$ Cephei star by our colleague Aerts and her coworkers (Aerts et al., 1992) and has now been used by several researchers (Mantegazza et al., 1994; Aerts \& Krisciunas, 1996). I presume that we shall hear more about the identification of pulsation modes during this colloquium.

Paul Ledoux was born in August 1914 in Forrières, a village in the Belgian Ardennes. After remarkable secondary-school studies, he entered the University of Liège in 1933, where he studied physics and graduated with the highest honours in 1937. Paul Ledoux' interest in astrophysics was awaked by the, at the time, young Prof. Pol Swings, who testified later that one had not to be a great prophet to foresee that Paul Ledoux would become a brilliant scientist.

After his studies and military service, Paul Ledoux, giving preference to theoretical research, went to the Institute of Theoretical Astrophysics in Oslo, where Prof. S. Rosseland introduced him into the subject of the radial oscillations and the stability of the stars. There he also published his first paper on the theory of stellar oscillations in the Astrophysica Norvegica (Ledoux, 1940).

In May 1940, Paul Ledoux left Oslo because of the invasion of the German Army in Norway and went to the Stockholm Observatory, where he stayed until November. Then he undertook a long journey through Russia and Siberia and, after various tribulations, reached the Yerkes Observatory of the University of Chicago in December 1940 (Swings, 1989).

Although it lasted only about one year, Paul Ledoux' stay at Oslo has had a decisive influence upon his scientific career. From that time on, he would dedicate his best energies mainly to the field of stellar oscillations and stellar stability. In the introduction of his review article entitled "Stellar Stability", which was also published in the Handbuch der Physik (Ledoux, 1958), he specified the main purposes of the study of stellar stability as follows: 
The most immediate purpose of the study of stellar stability is to discover the sources of the incipient instabilities which must be responsible for the observed variability of a great number of stars ...

Apart from the interpretation of variable stars, the study of stability has a more general meaning for the problem of stellar structure since any stability criterion may be looked upon as a necessary condition to be satisfied by any hydrostatic model.

At the Yerkes Observatory, Paul Ledoux began a long-lasting and fruitful association with Chandrasekhar and published some of the results of his research in Oslo and Stockholm. In a paper in collaboration with Pekeris (Ledoux \& Pekeris, 1941), he first applied variational methods for the determination of the fundamental radial oscillation mode of a star. In another paper (Ledoux, 1941), he showed that a main-sequence star with a mass larger than a critical mass of the order of $100 M_{\odot}$ becomes vibrationally or pulsationally unstable, that is, the amplitude of an harmonic oscillation increases exponentially in time since the driving processes in the core that are related to the nuclear-energy sources overcome the damping due to the energy transfer by radiation. Paul Ledoux' conjecture was that this instability would lead to finite oscillations, shock waves in the external layers, and mass loss. His result was confirmed and improved by Schwarzschild and Härm in 1959, who repeated Ledoux' computations "on the basis of ... detailed models for massive stars" and did so "not only for homogeneous models appropriate for the initial main sequence but also for inhomogeneous models representing subsequent evolution phases". In the 1970 's, numerical studies of the nonlinear effects showed that a star undergoes a swelling and mass losses.

Paul Ledoux' stay at the Yerkes Observatory was also of short duration. In September 1941, he left his family in the United States and joined the Belgian Armed Forces in Great Britain. Later he served within the Meteorological Section of the Royal Air Force (R.A.F.) and ended the war in the former Belgian Congo. There, he took advantage of his spare time to take up again his research on stellar stability, and conceived the idea of applying the virial theorem to the radial oscillations of stars. This application led to a simple derivation of the approximate formula for the period of the fundamental radial mode he had derived before by means of a variational method. He also derived a formula for the period in the case in which the star is in a steady uniform rotation. He sent a manuscript about his work to Chandrasekhar. The latter found Ledoux' piece of work highly interesting and, as not to lose time, took the initiative of submitting it to the Astrophysical Journal for publication. The paper appeared in 1945 with Paul Ledoux as author and the R.A.F., Stanleyville (today Kisangani), in the Belgian Congo, as the author's affiliation (Ledoux, 1945).

The virial approach has subsequently been extended to other problems of stellar stability. Chandrasekhar \& Fermi (1953) used it to take into account the effects of a magnetic field. Virial equations of higher orders were derived and applied to stability analyses of equilibrium configurations by Chandrasekhar and collaborators (see Chandrasekhar's book entitled Ellipsoidal Figures of Equilibrium, 1969).

Demobilized in September 1945, Paul Ledoux got his PhD degree at the University of Liège, went back to the Yerkes Observatory, where he rejoined 
his family, and stayed there for one year (Swings, 1989). Among the first, he examined the effects of changes of chemical composition on the stellar structure that are built up by the thermonuclear reactions in a star's core. He particularly considered the, at that time, new type of difficulty which arises at the boundary between a convective core and a radiative envelope of a main-sequence star as the mean molecular weight increases in the convective core because of the hydrogen-burning and remains unchanged in the radiative envelope. In his study, Paul Ledoux introduced a generalized form of the Schwarzschild criterion for convective stability in which the effect of a spatial variation in the chemical composition on a mass element moving with an unvarying chemical composition is taken into account. This criterion is now referred to as the Ledoux criterion and can be written in the general form

$$
\frac{d \ln T}{d \ln P} \leq\left(\frac{d \ln T}{d \ln P}\right)_{\mathrm{ad}}-\frac{\chi_{\bar{\mu}}}{\chi_{T}} \frac{d \ln \bar{\mu}}{d \ln P}
$$

where

$$
\chi_{\bar{\mu}}=\left(\frac{\partial \ln P}{\partial \ln \bar{\mu}}\right)_{\rho, T}, \quad \chi_{T}=\left(\frac{\partial \ln P}{\partial \ln T}\right)_{\rho, \bar{\mu}} .
$$

Paul Ledoux also introduced the notion of turbulent mixing that leads to the building up of a transition zone between the convective core and the radiative envelope with a varying mean molecular weight $\bar{\mu}$. In his paper of 1947 , he stated

... when $\bar{\mu}$ is assumed to change discontinuously at a point . ., turbulence in a small region surrounding that point will immediately result. This resulting turbulence will lead to a small amount of mixing of the internal and the external parts. And ... a neighboring stable state can be reached in consequence of this turbulence ...

Such transition zones are nowadays known as semi-convection zones and are considered to be present in many stars.

This important discovery has been a basis for his Thèse d'Agrégation pour l'Enseignement Supérieur, which he presented in 1949 at the University of Liège (Ledoux, 1949). After the acceptance of this thesis, Paul Ledoux' academic career really began. He became full professor in 1959 .

With one of his first students, Paul Ledoux considered the energy generation by the carbon cycle and the proton-proton reaction in white dwarfs. $\mathrm{He}$ showed that white dwarfs are vibrationally unstable with respect to these nuclear reactions, so that the presence of hydrogen in the internal regions of white dwarfs must be excluded (Ledoux \& Sauvenier-Goffin, 1950).

With another student, the late Arsène Boury, he studied the vibrational stability of stars that are originally composed of hydrogen or of a mixture of hydrogen and helium, and he discussed the implications of it for the possible enrichment of interstellar matter by helium produced in stars (Ledoux \& Boury, 1959).

Paul Ledoux devoted a great deal of attention to the study of the nonradial oscillations of gaseous stars and their complex spectra of eigenfrequencies. It is one of his very special merits that he realized the importance of nonradial 
oscillations in stellar stability and foresaw that the progress of observational techniques would reveal the presence of such oscillations in variable stars and close binaries. In an introductory lecture on nonradial oscillations delivered in 1974 in Canberra, Australia, he stated (Ledoux, 1974):

There has been lately quite a renewal of interest in the response of stars to nonradial perturbations aroused either by attempts at interpreting some types of variable stars like the $\beta$ Canis Majoris stars and the new white dwarf variables, or by phenomena in the external layers of the Sun like the 5 -min oscillation discovered by Leighton, or by the hope to add somewhat to our knowledge of convection and its penetration in nearby convectively stable zones, or by the desire to explore some new aspects of stellar stability which may be of great importance for the evolution of the star. On the other hand, one must expect that such non-radial motions should be easily excited in a variety of close double stars with eccentric orbits and it is likely that, with the extraordinary progress in observational techniques, these should become observable and be identified as such pretty soon. Finally there is a direct evidence in novae, perhaps even in planetary nebulae, for the presence of non-radial velocity fields.

Nearly at the time of the beginning of Paul Ledoux' scientific career, two important papers had been published on the nonradial oscillations of compressible masses in hydrostatic equilibrium. The first paper was by Pekeris (1938) on the nonradial oscillations of the compressible equilibrium sphere with uniform mass density, often denoted shortly as the compressible homogeneous model. The model cannot directly be applied to stars but has served to illustrate some properties of the general problem. Pekeris had shown that, for each degree $\ell$ of the spherical harmonic, two spectra of eigenvalues exist for the square of the angular frequency: one spectrum of positive eigenvalues increasing indefinitely with the radial order of the mode, and another spectrum of negative eigenvalues tending towards zero as the radial order of the mode increases.

The second important paper was by Cowling (1941) on the nonradial oscillations of polytropic models. By neglecting the Eulerian perturbation of the gravitational potential, as was already done before by Emden (1907), and referring to analogies with the eigenvalue problems of the Sturm-Liouville type, Cowling had come to conclusions partly similar to the results of Pekeris with regard to the homogeneous model: for each degree $\ell$ of the spherical harmonic, the eigenvalue problem of the nonradial oscillations of the polytropic models admits of two spectra of eigenvalues for the square of the angular frequency; one spectrum consists of indefinitely increasing eigenvalues, and the other spectrum, of eigenvalues decreasing towards zero.

A first point of difference was that, for the particular case of the polytropic model with index $n=3$, for which Cowling had made some calculations, the eigenvalues of both spectra are positive. In his paper, Cowling had called the modes associated with the eigenvalues of the first spectrum pressure modes or $p$-modes, and the modes associated with the eigenvalues of the second spectrum, gravity modes or $g$-modes.

Another point of difference was that, for $\ell \geq 2$, Cowling had distinguished a fundamental mode or $f$-mode which is associated with an eigenvalue situated 
between the eigenvalues for the $p$-modes and those for the $g$-modes. The mode is characterized by the property that the radial component of the Lagrangian displacement and the Eulerian perturbation of the density keep the same sign along each radius of the model.

Paul Ledoux studied the papers by Pekeris and Cowling very carefully (see Sauvenier-Goffin 1951) and laid himself out to establish a closer link between them. The modes of the compressible homogeneous model that are associated with positive eigenvalues for the square of the angular frequency could manifestly be identified as Cowling's $p$-modes. However, the interpretation of the modes of the compressible homogeneous model that are associated with negative eigenvalues for the square of the angular frequency and that are dynamically unstable was less obvious. Paul Ledoux related the unstable character of the modes to the violation of Schwarzschild's criterion for local convective stability in the equilibrium model; he interpreted the dynamically unstable modes, in the homogeneous model and in other models as well, as $g$-modes which render the starting convective motions in superadiabatic regions of these models (see Ledoux \& Walraven, 1958, Section 76; Ledoux, 1958, Section 16). In his Thèse d'Agrégation pour l'Enseignement Supérieur, he observed:

Il semble ... que sous son aspect le plus général, la question de la stabilité dynamique se rattache à celle de la stabilité vis-à-vis des courants de convection à laquelle est associée le critère de K. Schwarzschild. $^{1}$

Paul Ledoux was probably the first to stress, in such an explicit way, the connection between the global dynamic instability of a star through $g$-modes and the local convective instability (Ledoux, 1949, Section 3.4).

For a certain time, another intriguing question was the apparent absence of $f$-modes in the compressible homogeneous model. This absence did raise some doubts about Cowling's identification of the $f$-modes as modes distinct from the $p$ - and the $g$-modes. The matter was clarified in 1964 when Chandrasekhar showed, by the use of a variational principle (see also Chandrasekhar \& Lebovitz, 1964), that the modes of the incompressible homogeneous model that had been determined by Kelvin one century before (Thomson, 1863) are also modes of the compressible homogeneous model. It then became clear that Cowling's $f$ modes are the transpositions of the Kelvin modes of the homogeneous model to the models with density stratifications and that they have a character definitely different from that of the $p$-and the $g$-modes. In particular, the $f$-modes associated with $\ell=1$, which are time-independent, represent uniform translations of the equilibrium star and are the only admissible divergence-free modes in a model with density stratification (Robe, 1965).

Under Paul Ledoux' stimulating guidance, Cowling's classification of the nonradial oscillations was confirmed for physical models of massive stars composed of a convective core in adiabatic equilibrium and a radiative envelope (Smeyers, 1967). In these models, the $g$-modes are all dynamically stable. An interesting question raised by Ledoux was what influence the introduction of

${ }^{1}$ It seems ... that in its most general form the question of dynamic stability is related to the stability with respect to convective flows, with which the criterion of $\mathrm{K}$. Schwarzschild is associated. 
a slight superadiabatic gradient of temperature into the convective core would have on the $g$-modes. Dynamically unstable $g$-modes were expected to arise, but it was unclear how this would occur. The question was resolved both on the basis of numerical computations for an artificial model and on the basis of an analogy with the eigenvalue problems of the Sturm-Liouville type. It resulted that, in stars containing one or more superadiabatic regions, each $g$-spectrum of eigenvalues is decomposed into a spectrum of positive eigenvalues and a spectrum of negative eigenvalues. The $g$-modes associated with positive eigenvalues were denominated $g^{+}$-modes and are mainly related to the convectively stable regions, while the $g$-modes associated with negative eigenvalues were denominated $g^{-}$-modes and are mainly related to the convectively unstable regions (Ledoux \& Smeyers, 1966).

On the same line of thought, Lebovitz (1966) showed that Schwarzschild's criterion for convective stability is not only a sufficient but also a necessary condition for a star to be dynamically stable. Lebovitz' proof implies that $g^{-}$modes exist in any stellar model that contains a superadiabatic zone, however small this zone may be. It also fully confirmed Paul Ledoux' earlier view on the connection between the global dynamic instability of a star with respect to $g$-modes and the local convective instabilities in the same star.

A next step forwards in the study of the nonradial oscillations of stars under Paul Ledoux' guidance consisted of a series of investigations on the effect of the central condensation of stellar models on the numbers of nodes in the eigenfunctions of lower-order modes. Robe pointed out that, in centrally condensed polytropes, the $f$-mode and the lowest-order $p$-and $g$-modes gain additional nodes (Robe, 1968; Ledoux, 1969; Ledoux, 1974). A regularity in the numbers of nodes was observed by Scuflaire (1974). Later on, the anomalous numbers of nodes found for lower-order modes in centrally condensed models were shown to be related to phenomena of mode bumping or avoided crossings of $g^{+}$-modes with the $f$-mode and the lowest-order $p$-modes of the same degree. Avoided crossings occur in models with a higher central condensation of the mass, since the eigenfrequencies of the $g^{+}$-modes increase while those of the $f$-mode and the $p$-modes remain almost unchanged (Osaki, 1975; Shibahashi \& Osaki, 1976; Aizenman et al., 1977).

In addition to this, the set of admissible normal modes of a gaseous star in hydrostatic equilibrium was complemented by the toroidal modes as a result of a group-theoretical study by Paul Ledoux' collaborator Perdang (1968). Although they seem to be trivial modes at first sight, toroidal modes are essential for the description of motions in a star with vorticity around the local normal to the equipotential surface.

Another special scientific merit of Paul Ledoux is that he opened the field of the asymptotic representations of stellar oscillations in 1962 by developing an asymptotic representation of higher-order radial oscillations of a star (Ledoux, 1962; Ledoux, 1963). He soon tried to extend his investigation to low-degree, higher-order nonradial oscillations with the collaboration of a student (Iweins, 1964). His aim was to apply the same asymptotic method either to a secondorder differential equation in the radial component of the Lagrangian displacement or to a second-order differential equation in the Eulerian pressure perturbation. Both differential equations were derived in the Cowling approximation. 
However, both differential equations have a singularity whose position along the radius depends on the eigenfrequency of the mode considered and is called therefore a mobile singularity. In order to avoid these mobile singularities, Ledoux proposed to use one differential equation from the star's centre and the other one from the star's surface, and to join the resulting asymptotic solutions at a point of their common domain of validity. To Ledoux' regret, this work was never published despite its importance. The same procedure has been rediscovered a few years later by Vandakurov (1967) and has been used by other investigators for many years. I especially refer to Tassoul's (1980) second-order asymptotic theory for low-degree higher-order $p$ - and $g$-modes in the Cowling approximation.

The field of the asymptotic representation of stellar oscillations is well developed at the present time. Monique Tassoul has made major progress in 1990 by returning to the use of a system of differential equations in the divergence and the radial component of the Lagrangian displacement established earlier by Pekeris (1938). From then on, one no longer needs to adopt the Cowling approximation, nor is one any longer confronted with mobile singularites in the equations. Furthermore, boundary-layer techniques and multiple variable expansion procedures have proven to be adequate for the representation of $p$ - and $g^{+}$-modes as standing waves that originate from waves propagating to-and-fro in a cavity inside the star (Smeyers et al., 1995, 1996). A still major unsettled question remains the derivation of asymptotic solutions from the star's surface that are not based on the assumption that the mass density is an analytic function near that surface. For the small frequency separations of low-degree, high frequency $p$-modes, an alternative description based on a generalization of the first Born approximation for the scattering of acoustic waves modified by buoyancy and gravity in the stellar core has been developed by Roxburgh \& Vorontsov (1994).

Today we all realize that the theory of the nonradial oscillations of stars has been fundamental for the identification of solar 5-min oscillations as global $p$-modes of higher degrees and low radial orders (Deubner, 1975) and for the development of the helioseismology (see, e.g., Deubner \& Gough, 1984).

Paul Ledoux also stressed the importance of the secular or thermal stability besides that of the dynamic and the vibrational stability. In a review published in 1965 (Ledoux, 1965), he noted:

While the problems of dynamical and vibrational stability have been the object of extensive studies that led to precise criteria, secular stability has been rather neglected up to now. Its discussion seems to stem from a remark by H.N. Russell a long time ago, when very little was known of nuclear reactions in stellar interiors except that they should provide a much longer lifetime for ordinary stars than that allowed by the Helmholtz-Kelvin contraction hypothesis.

Already at the beginning of the 1960's, he was aware of a number of delicate problems that might arise in computations of stellar evolution and emphasized the necessity of supplementing these computations at some phases of the evolution by studies of secular stability (Ledoux, 1960). In his review of 1965, he also noted: 
With the increasing importance of evolutionary considerations, the need for an adequate solution to the general problem of secular stability will probably be felt more urgently. In fact, it is on this basis that the method of treating stellar evolution (as a series of equilibrium or quasi-equilibrium configurations), the type of time derivatives to be kept, the time step to be adopted, should be justified. It may also throw light on particularly complex phases of stellar evolution when the nonlinear character of the general problem and error feedback in the time derivatives makes it difficult ..., starting from a given quasi-equilibrium solution, to determine the one immediately following after a finite time step. Furthermore, there is always a possibility that, in continuing a series of models by finite steps, one might overpass the critical point where stability is lost without noticing it, at least for some time.

In all these respects, a comparison with the classical problem of the evolution of a mechanical system depending on a slowly varying parameter might be of interest. In that case the theory of linear series of equilibrium configurations and of exchange or loss of stability at their bifurcation, turning, or terminating points as developed by Poincaré ... yields a global view of the stability problem which, for instance, has proved very useful in the discussion of the evolution of an incompressible mass in solid rotation with a slowly increasing angular velocity.

As a final note on Paul Ledoux' scientific merits, I may draw the attention to a point that again concerns the influence of a uniform axial rotation on nonradial stellar oscillations. In 1978, Papaloizou \& Pringle showed that the introduction of a uniform axial rotation generates a new set of modes besides the known sets of $p_{-}, g_{-}$, and $f$-modes. At the first order of approximation, the modes have angular frequencies proportional to the angular frequency of rotation $\Omega$ and are independent of the star's structure. They were denoted rotation modes or $r$-modes by Papaloizou \& Pringle and originate from purely toroidal time-independent displacement fields in the nonrotating equilibrium star. With regard to the discovery of the $r$-modes in rotating stars, Morris Aizenman (1980) had the following comment on a passage in the review article on variable stars by Ledoux \& Walraven published in the Handbuch der Physik (Ledoux \& Walraven, 1958, under Eq. (82.20)):

It is interesting to note a comment made by Ledoux (1958). In analyzing the results of the compressible [homogeneous] model, he [Ledoux] stated that for the axially symmetric mode there is an additional solution which is directly proportional to the angular rotation frequency. He discarded this solution as spurious and referred to an earlier analysis (Ledoux, 1949). The spurious solution is, in fact, the toroidal solution obtained by Papaloizou and Pringle. The result Ledoux obtained in 1949 is consistent with that of Papaloizou and Pringle because the solution is independent of [the star's] structure through terms including $\Omega^{2}$.

The earlier analysis referred to is in Paul Ledoux' Thèse d'Agrégation pour l'Enseignement Supérieur. 
In conclusion, Paul Ledoux has been a scientist of great stature. One is struck by the number of questions of stellar stability he thought deeply about. He played a substantial role in establishing a solid foundation for the theory of stellar stability. Paul Ledoux' mastery of the field of stellar stability has found his clearest expression in the numerous reviews of the subject written by him and especially in the two remarkable reviews published in Volume 51 of the Handbuch der Physik (1958), which have been authoritative for a very long time.

Paul Ledoux was not only an outstanding scientist, he was also a wise and high-minded person, modest with regard to his own accomplishments, and always affable, in harmony with his family name.

\section{References}

Aerts, C., De Pauw, M., \& Waelkens, C. 1992, A\&A, 266, 294

Aerts, C. \& Krisciunas, K. 1996, MNRAS, 278, 877

Aizenman, M. L. 1980, in Lecture Notes in Physics, 125, Nonradial and Nonlinear Stellar Pulsation, ed. H. A. Hill \& W. A. Dziembowski (Berlin: Springer), 76

Aizenman, M., Smeyers, P., \& Weigert, A. 1977, A\&A, 58, 41

Balona, L. 1986a, MNRAS, 219, 111

Balona, L. 1986b , MNRAS, 220, 647

Balona, L. 1987, MNRAS, 224, 41

Chandrasekhar, S. 1964, ApJ, 139, 664

Chandrasekhar, S. 1969, Ellipsoidal Figures of Equilibrium (New Haven: Yale University Press)

Chandrasekhar, S., \& Fermi, E. 1953, ApJ, 118, 116

Chandrasekhar, S., \& Lebovitz, N.R. 1964, ApJ, 140, 1517

Cowling, T. G. 1941, MNRAS, 101, 367

Denis, J. 1972, A\&A, 20, 151

Deubner, F.-L. 1975, A\&A, 44, 371

Deubner, F.-L. \& Gough, D. 1984, ARA\&A, 22, 593

Emden, R. 1907, Gaskugeln (Leipzig: Teubner)

Iweins, P. 1964, Mémoire de Licence, Université de Liège

Lebovitz, N. R. 1966, ApJ, 146, 946

Ledoux, P. 1940, Astrophys. Norvegica, 3, No. 8, 193

Ledoux, P. 1941, ApJ, 94, 537

Ledoux, P. 1945, ApJ, 102, 143

Ledoux, P. 1947, ApJ, 105, 305

Ledoux, P. 1949, Mém. Soc. Roy. Sci. Liège, 4ème Série, T. IX, 1

Ledoux, P. 1951, ApJ, 114, 373

Ledoux, P. 1958, in Handbuch der Physik, 51, Astrophysik II: Sternaufbau, ed. S. Flügge (Berlin: Springer), 605

Ledoux, P. 1960, Bull. Acad. Roy. Belg., Cl. Sci., 46, 429

Ledoux, P. 1962, Bull. Acad. Roy. Belg., Cl. Sci., 48, 240

Ledoux, P. 1963, Bull. Acad. Roy. Belg., Cl. Sci., 49, 286 
Ledoux, P. 1965, in Stars and Stellar Systems, 8, Stellar Structure, ed. L. H. Aller \& D. B. McLaughlin, (Chicago: University of Chicago Press), 499

Ledoux, P. 1969, in Cours de Perfectionnement de l'Association Vaudoise des Chercheurs en Physique, 11, La Structure Interne des Etoiles, ed. C. Joseph, G. Janin, A. Maeder, M. Mayor (Geneva: Geneva Observatory), 44

Ledoux, P. 1974, in IAU Symp. 59, Stellar Instability and Evolution, ed. P. Ledoux, A. Noels, \& A. W. Rodgers, 135

Ledoux, P. \& Boury, A. 1959, Mém. Soc. Roy. Sci. Liège, 5ème Série, 3, 298

Ledoux, P. \& Pekeris, C. L. 1941, ApJ, 94, 124

Ledoux, P. \& Renson, P. 1967, ARA\&A, 4, 293

Ledoux, P. \& Sauvenier-Goffin, E. 1950, ApJ, 111, 611

Ledoux, P. \& Smeyers, P. 1966, C. R. Acad. Sc. Paris, 262, 841

Ledoux, P. \& Walraven, Th. 1958, in Handbuch der Physik, 51, Astrophysik II: Sternaufbau, ed. S. Flügge (Berlin: Springer), 353

Mantegazza, L., Poretti, E., \& Bossi, M. 1994, A\&A, 287, 95

Osaki, Y. 1971, PASJ, 23, 485

Osaki, Y. 1975, PASJ, 27, 237

Papaloizou, J. \& Pringle, J.E. 1978, MNRAS, 182, 423

Pekeris, C. L. 1938, ApJ, 88, 189

Perdang, J. 1968, Ap\&SS, 1, 355

Robe, H. 1965, Bull. Acad. Roy. Belge, Cl. Sc., 51, 598

Robe, H. 1968, Thèse de Doctorat, Université de Liège

Roxburgh, I. W. \& Vorontsov, S. V. 1994, MNRAS, 267, 297

Saio, H. 1981, ApJ, 244, 299

Sauvenier-Goffin, E. 1951, Bull. Soc. Roy. Sci. Liège 20, 20

Schwarzschild, M. \& Härm, R. 1959, ApJ, 129, 637

Scuflaire, R. 1974, A\&A, 36, 107

Shibahashi, H. \& Osaki, Y. 1976, PASJ, 28, 199

Simon, R. 1969, A\&A, 2, 390

Smeyers, P. 1967, Bull. Soc. Roy. Sci. Liège, 36, 357

Smeyers, P., De Boeck, I., Van Hoolst, T., \& Decock, L. 1995, A\&A, 301, 105

Smeyers, P. \& Denis, J. 1971, A\&A, 14, 311

Smeyers, P., Vansimpsen, T., De Boeck, I., \& Van Hoolst, T. 1996, A\&A, 307, 105

Struve, O. 1950, ApJ, 112, 520

Swings, J.-P. 1989, Ap\&SS, 155, 179

Tassoul, M. 1980, ApJS, 43, 469

Tassoul, M. 1990, ApJ, 358, 313

Thomson, W. 1863, Phil. Trans. Roy. Soc. London, 153, 583

Unno, W., Osaki, Y., Ando, H., Saio, H., \& Shibahashi, H. 1989, Nonradial Oscillations of Stars (University of Tokyo Press)

Vandakurov, Yu. V. 1967, AZh, 44, 876 